\title{
Biobjective Optimization-Based Frequency Regulation of Power Grids with High-Participated Renewable Energy and Energy Storage Systems
}

\author{
Tingyi He, ${ }^{1}$ Shengnan $\mathrm{Li}^{1}{ }^{1}$ Shuijun $\mathrm{Wu},{ }^{1}$ Chuangzhi $\mathrm{Li}^{2}$ and Biao $\mathrm{Xu} \mathbb{D}^{2}$ \\ ${ }^{1}$ Yunnan Power Grid Co., Ltd., Electric Power Research Institute, Kunming 650200, China \\ ${ }^{2}$ College of Engineering, Shantou University, Shantou 515063, China \\ Correspondence should be addressed to Biao Xu; xubiao@stu.edu.cn
}

Received 3 February 2021; Revised 16 February 2021; Accepted 23 February 2021; Published 1 March 2021

Academic Editor: Bo Yang

Copyright (c) 2021 Tingyi He et al. This is an open access article distributed under the Creative Commons Attribution License, which permits unrestricted use, distribution, and reproduction in any medium, provided the original work is properly cited.

\begin{abstract}
Large-scale renewable energy sources connected to the grid bring new problems and challenges to the automatic generation control (AGC) of the power system. In order to improve the dynamic response performance of AGC, a biobjective of complementary control (BOCC) with high-participation of energy storage resources (ESRs) is established, with the minimization of total power deviation and the minimization of regulation mileage payment. To address this problem, the strength Pareto evolutionary algorithm is employed to quickly acquire a high-quality Pareto front for BOCC. Based on the entropy weight method (EWM), grey target decision-making theory is designed to choose a compromise dispatch scheme that takes both of the operating economy and power quality into account. At last, an extended two-area load frequency control (LFC) model with seven AGC units is taken to verify the effectiveness and the performance of the proposed method.
\end{abstract}

\section{Introduction}

Automatic generation control (AGC) is one of the important tools to maintain the contact line exchange power and realtime network frequency of the power system within the schedule when the grid experiences load disturbances [1]. Traditional AGC units mainly include thermal and hydro units, which are hard to fast track the dynamic power input commands due to their low regulation performance [2]. With the development of renewable energy, a large number of wind power and photovoltaic $(\mathrm{PV})$ units are connected to the grid. On the one hand, as the outputs of wind power and PV units are regulated by power electronics equipment, they can quickly respond to the dynamic power input regulation commands. On the other hand, since the large-scale wind power and $\mathrm{PV}$ units are subject to climate conditions, their generation outputs have large random fluctuations, which aggravates the pressure on frequency regulation of power system. In addition, more and more new energy storage resources (ESRs) are joining the grid, such as chemical battery energy storage, electric vehicles, and grid-scale battery storage.

Currently, there is a worldwide effort to balance the intermittency of renewable energy in the grid with highcapacity batteries, in which these ESRs have considerably faster regulation performance than conventional power generators. In China, a vast $200 \mathrm{MW}, 800 \mathrm{MWh}$ Vanadium Redox Flow Battery in the Dalian High-Tech zone is currently being readied for operation, which is the largest chemical energy storage plant in the world [3]. In California, the largest battery energy storage project in the world, Gateway Energy Storage, is in progress [4]. The addition of energy storage can improve the economic operation of the system. It can help reduce the pressure on frequency regulation of power system caused by PV units. Wind power and PV units generate electricity by storing excess energy in large-capacity battery sets, which are fed back into the grid when the batteries are not generating electricity to relieve the pressure on frequency regulation of the power system. 
Besides, the traditional frequency regulation compensation scheme does not effectively stimulate fast response resources to be engaged in AGC because it fails to provide fair compensation in terms of actual regulation performance [5]. The Federal Energy Regulatory Commission (FERC) published Order No. 755 in 2011 to establish a more reasonably priced compensation framework [6]. This command document showed that the compensation payment in the performance-based frequency regulation market is not only determined by the regulation capacity, but also by the actual regulation volume and unit performance [7]. However, some independent system operators (ISOs) implemented 755 negatively and did not establish fair and dynamic markets, because they adopted existing rules that protect existing resources such as liquefied natural gas (LNG) units from competition that is created by ESRs with lower regulation mileage payment and faster regulation performance. And then in February 2018, the FERC issued a landmark order for ESRs, Order No. 841, setting standards for ESR participation in frequency regulation [8]. The regulation units such as ESRs with higher regulation performance will be preferred for ISOs when receiving the same power regulation command. And it will be paid more compared to the regulation unit with lower regulation performance.

So far, few research studies have addressed the complementary control between wind, PV, ESRs, and other frequency regulation resources. In [9], a new biobjective optimization model of real-time AGC dispatch (BOAD) was constructed, with the minimization of the total power deviation and the regulation mileage payment. However, it did not consider ESRs to participate in AGC. Hence, this paper presents a new biobjective of complementary control (BOCC) for AGC with the high participation of ESRs.

To solve the BOCC with high nonlinearity, the metaheuristic-based multiobjective optimization algorithms show stronger global searching ability than the traditional mathematical optimization methods [10-15]. Widely used multiobjective algorithms include the improved strength Pareto evolutionary algorithm (SPEA2) [16] and a nondominated sorting genetic algorithm II (NSGA-II) [17]. Compared with NSGA-II, the fitness function of SPEA2 is not calculated directly from the value of the objective function, which is indirectly linked to the value of the objective function but to the Pareto strength. SPEA2 ensures that the converged solution set is a set of noninferior solutions and considers the density of individuals in the solution space to maintain the diversity of the population and ensure the uniform distribution of the solution set. Therefore, this paper adopts SPEA2 to solve BOCC.

In addition, since ISOs can allocate only one AGC dispatch signal to each unit at each control interval, the ISOs should select the best suitable solution from the multiple solutions obtained on the Pareto front. This requires the application of a decision-making method to help the ISOs select a best compromise dispatch scheme from the set of obtained Pareto solutions. Grey target decision-making is one of the methods to solve multiobjective optimization by using the grey systematic theory to select a best compromise solution. In [18], a transformation operator of rewarding the good and punishing the bad was proposed to classify the index of effect sample matrix into three categories. Furthermore, some subjective weighting methods such as the analytic hierarchy process method (AHP) [19] and Delphi method [20] are usually used to determine the weights of indexes by the experts' evaluation matrix, but they easily lead to deviations in indicator weights, while the objective weighting method such as the entropy weight method (EWM) is based on inherent information of decisionmaking matric to determine the weights of indexes. And then grey target decision-making theory with EWM is designed to choose a dispatch plan that takes into account both of the operating economy and power quality, which can provide an objective decision without a subjective judgment.

The remaining of this work is organized as follows: Section 2 presents the mathematical model of BOCC. Section 3 gives the detailed implementation of SPEA2 and grey target decision-making with EWM for BOCC. The simulation results and discussions are given in Section 4. Finally, Section 5 concludes the paper.

\section{Mathematical Model of BOCC}

2.1. AGC Dispatch Framework. AGC mainly consists of two operations. Figure 1 displays an extended two-area load frequency control (LFC) model. $\Delta P_{T}$ is the junction line power exchange deviation; $\Delta f$ is the real-time frequency deviation; $\Delta P_{\text {out }}$ is the actual regulated power output; and $\Delta P_{D}$ is the power disturbance. The goal of the first operation is to approximate the real-time power disturbance by a PI controller with the inputs of the real-time frequency deviation $\Delta f$ and the junction line power exchange deviation $\Delta P_{T}$. In the second operation, the ISO assigns the total generation command $\Delta P_{C}$ to all the AGC units. Note that this paper focuses on the specific allocation process of the second operation, which is addressed by the proposed SPEA2 and grey target decision-making with EWM. The specific framework of the AGC dispatch model has been given in reference [9], and the ESRs are added to participate in the AGC scheduling process.

2.2. Constraints. In BOCC, it should consider various constraints, including the power balance constraint, dynamic response process with generation ramp constraint (GRC), regulation capacity constraint, and energy transfer constraint [9], as follows:

(1) Power balance constraint: at the $k^{\text {th }}$ control interval, the total power regulation command output by the controller should be equal to the sum of the power regulation input signals received by all AGC units, as follows:

$$
\sum_{i=1}^{n} \Delta P_{i}^{\mathrm{in}}(k)-\Delta P_{c}(k)=0
$$

where $\Delta P_{i}^{\text {in }}(k)$ denotes the input power command received by the $i^{\text {th }}$ unit at $k^{\text {th }}$ control interval and $\Delta P_{c}(k)$ denotes the output of the PI controller. 


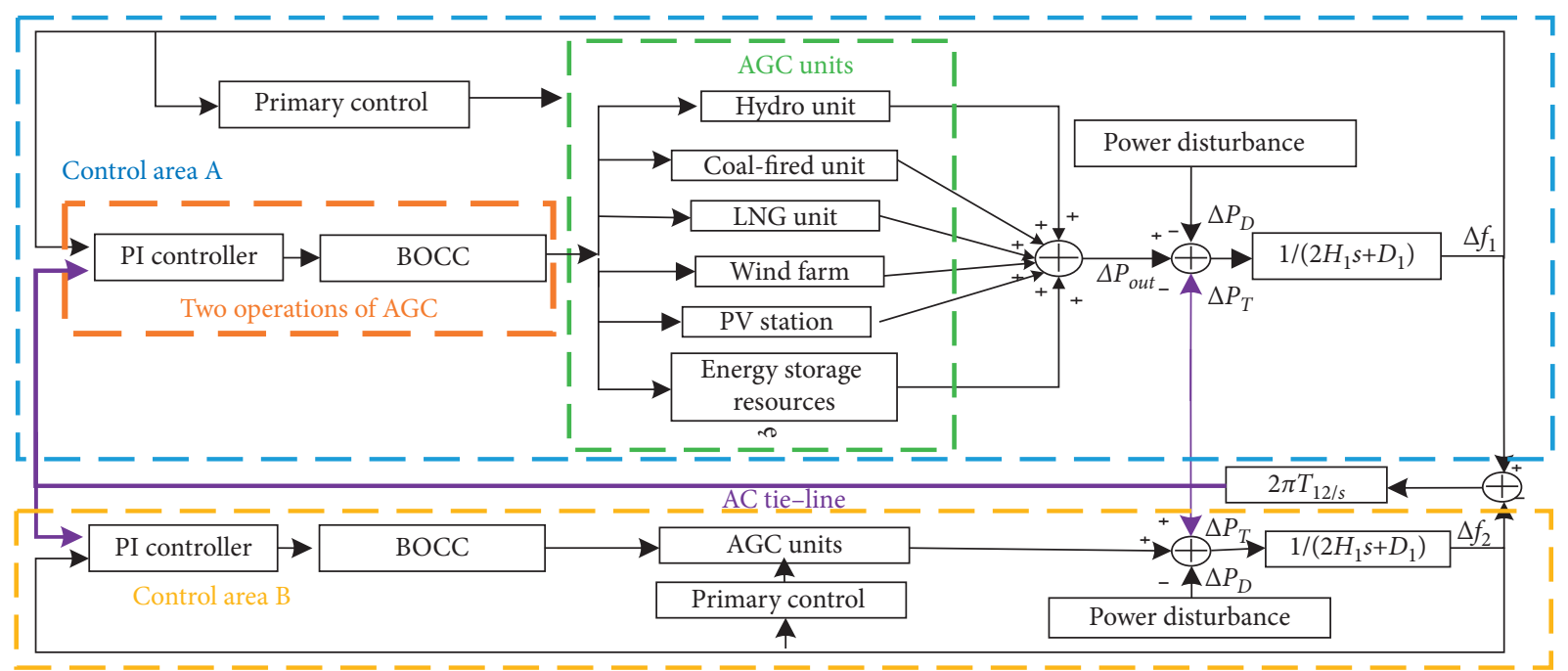

FIGURE 1: Framework of AGC on the extended two-area LFC model.

(2) GRC: depending on the response time delay, AGC units can be sorted into different types of units [21]. Like wind power and PV units, the dynamic response model of ESRs has no a generation ramp constraint (GRC), as shown in Table 1. And the dynamic response function is shown in Figure 2. Without considering the GRC and power limiter, the actual regulated power output is relating to a Laplacian inverse transfer function, as follows:

$$
M_{i}^{\text {in }}(k)=\Delta P_{i}^{\text {in }}(k)-P_{i}^{\text {in }}(k-1),
$$

where $G_{i}(s)$ is the energy transfer function of the $i^{\text {th }}$ AGC unit; $\Delta T$ is the delay time constant of the $i^{\text {th }}$ unit; and $M_{i}^{\text {in }}(k)$ is the regulation mileage input of the $i^{\text {th }}$ AGC unit at $k^{\text {th }}$ control interval.

If the GRC and power limiter are considered, then the output of the AGC unit can be calculated, as follows:

$$
\begin{gathered}
\Delta P_{i}^{\text {out }}(t)=L^{-1}\left\{\frac{G_{i}(s)}{s\left(1+T_{d}^{i} s\right)} \sum_{k=1}^{N}\left[e^{-\Delta T(k-1) s} M_{i}^{\text {in }}(k)\right]\right\} \\
\Delta P_{i}^{\text {out }}(k)=\Delta P_{i}^{\text {out }}(t=k \cdot \Delta T)
\end{gathered}
$$

$$
\begin{aligned}
\Delta P_{i}^{\text {out }}(k) & = \begin{cases}\Delta P_{i}^{\text {out }}(k-1)+R_{i}^{\text {min }}, \quad \text { if } \Delta P_{i}^{\text {out }}(k)<R_{i}^{\text {min }}, \\
\Delta P_{i}^{\text {out }}(k), \quad \text { if } R_{i}^{\text {min }} \leq \Delta P_{i}^{\text {out }}(k) \leq R_{i}^{\text {max }}, \\
\Delta P_{i}^{\text {out }}(k-1)+R_{i}^{\text {max }}, & \text { if } \Delta P_{i}^{\text {out }}(k)>R_{i}^{\text {min }},\end{cases} \\
R_{i}^{\text {min }} & = \begin{cases}0, & \text { if } M_{c}(k) \geq 0, \\
\max \left[-\Delta P_{i}^{\text {rate }} \cdot \Delta T, \Delta P_{i}^{\text {min }}-\Delta P_{i}^{\text {out }}(k-1)\right], & \text { if } M_{c}(k)<0,\end{cases} \\
R_{i}^{\max } & = \begin{cases}\min \left[\Delta P_{i}^{\text {rate }} \cdot \Delta T, \Delta P_{i}^{\max }-\Delta P_{i}^{\text {out }}(k-1)\right], & \text { if } M_{c}(k) \geq 0, \\
0, & \text { if } M_{c}(k)<0,\end{cases}
\end{aligned}
$$

where $\Delta P_{i}^{\min }$ and $\Delta P_{i}^{\max }$ are the minimum and maximum regulation capacities of the $i^{\text {th }}$ unit, respectively; $R_{i}^{\min }$ and $R_{i}^{\max }$ are the minimum and maximum power regulation variations of the $i^{\text {th }}$ unit, respectively; and $\Delta P_{i}^{\text {rate }}$ is the maximum ramp rate of the $i^{\text {th }}$ unit.
2.3. Objective Function. Since the proposed BOCC is intended to minimize the total power deviation between the regulation command and the actual power regulation output and minimize the regulation mileage payment, the objective functions can be written as follows: 
TABLE 1: Types of transfer functions for various units.

\begin{tabular}{lc}
\hline Type & Transfer function $G(s)$ \\
\hline Non-reheat steam unit & $1 / 1+T_{1} s$ \\
Reheat steam unit & $1+T_{2} s /\left(1+T_{3} s\right)\left(1+T_{4} s\right)\left(1+T_{5} s\right)$ \\
Hydro unit & $\left(1-T_{6} s\right)\left(1+T_{7} s\right) /\left(1+0.5 T_{6} s\right)\left(1+T_{8} s\right)$ \\
WT and PV & $1 / 1+T_{9} s$ \\
ESRs & $1 / 1+T_{10} s$ \\
\hline
\end{tabular}

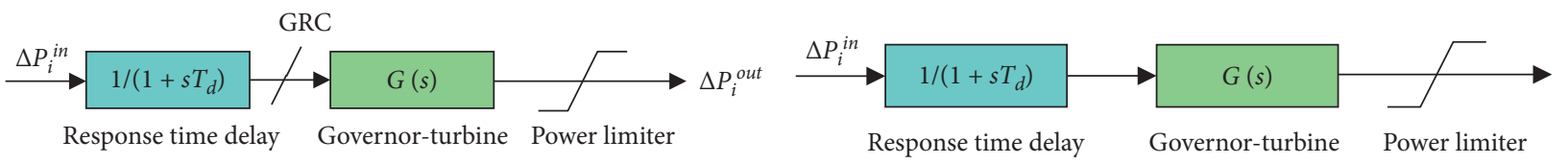

(a)

(b)

FIgURE 2: Dynamic response models: (a) conventional units; (b) renewable units and ESRs.

$$
\left\{\begin{array}{l}
\min f_{1}=\sum_{k=1}^{N}\left|\Delta P_{c}(k)-\sum_{i=1}^{n} \Delta P_{i}^{\text {out }}(k+1)\right| \\
\min f_{2}=\sum_{i=1}^{n} R_{i}
\end{array}\right.
$$

where $R_{i}$ represents the regulation mileage payment for the $i^{\text {th }}$ AGC unit, as follows:

$$
\begin{gathered}
R_{i}=\sum_{k=1}^{N} \gamma S_{i}^{P} M_{i}^{\text {out }}(k), \\
M_{i}^{\text {out }}(k)=\left|\Delta P_{i}^{\text {out }}(k)-\Delta P_{i}^{\text {out }}(k-1)\right|,
\end{gathered}
$$

where $\lambda$ is the price of regulation mileage; $S_{i}^{p}$ is the performance score; $\Delta P_{i}^{\text {out }}(k)$ is the actual regulation power output of $i^{\text {th }}$ unit at the $k^{\text {th }}$ time control interval; and $M_{i}^{\text {out }}(k)$ is the regulation mileage output at the $k$ th control interval.

\section{Design of SPEA2 and Grey Target Decision- Making for BOCC}

\subsection{Principle of SPEA2}

3.1.1. Optimization Operations. In general, SPEA2 mainly contains four operations, as follows:
(1) Initialization: generating a random set of solutions in the solution space $X_{j}=\Delta P_{i}^{\text {in }}(i=1,2, \ldots, n, j=$ $1,2, \ldots, N) X_{j}$ forms the initialized population $P_{0}$, and the size of the population is N. And then creating the empty archive $\mathbf{P}_{\mathbf{0}}^{\prime}=\varnothing$, and the size of the archive is $\bar{N}$. In addition, at the $k$ th control interval, the lower and upper boundary of all optimization variables are set equally to the lower and upper adjustment capacity of all units, respectively.

(2) Fitness calculation: based on the equation power balance constraint in (1), the variable $x_{d}=\Delta P_{c}(k)-$ $\sum_{i=1(i \neq d)}^{n} \Delta P_{i}^{\text {in }}(k)$ can be regarded as a known quantity during optimization. By considering the constraint of the $d^{\text {th }}$ unit output, if the boundary conditions are violated, the fitness function of $j^{\text {th }}$ individual should be given with a large penalty value. Hence, the fitness function can be designed as follows:

$$
\left\{\begin{array}{l}
F_{2}\left(X_{j}\right)=F_{1}\left(X_{j}\right)=\left\{\left[x_{d}-\Delta P_{d}^{\max }\right]\left[x_{d}-\Delta P_{d}^{\min }\right]+1\right\} \cdot 10^{8}, \quad \text { if } x_{d}>\Delta P_{d}^{\max } \text { or } x_{d}<\Delta P_{d}^{\min } \\
F_{1}\left(X_{j}\right)=f_{1}\left(X_{j}\right), F_{2}\left(X_{j}\right)=f_{2}\left(X_{j}\right), \quad \text { else }
\end{array}\right.
$$

where $\Delta P_{d}^{\min }$ and $\Delta P_{d}^{\max }$ are the minimum and maximum regulation capacities of the $d^{\text {th }}$ unit, respectively.

(3) Sorted by actual fitness function and copy: the actual fitness function can be calculated by equations (11)-(15). Then, the results can be sorted by adaptation value. When the number of noninferior solutions is less than the number of archive size $\bar{N}$, copy the first $\bar{N}-\left|\bar{P}_{\mathbf{t}+\mathbf{1}}\right|$ individuals $X_{j}$ with $F\left(X_{j}\right) \geq 1$ from the resulting ordered list to $\vec{P}_{\mathbf{t}+\mathbf{1}}[11]$, and $|\cdot|$ corresponds to the number of elements of a set.

(4) Archive truncation procedure: when the number of noninferior solutions exceeds the number of archive 
size $\bar{N}$, some of the solutions should be removed from $\bar{P}_{t+1}$ until $\left|\bar{P}_{t+1}\right|=\bar{N}$.

3.1.2. Parameters of SPEA2. (1) Strength Value $S\left(X_{i}\right)$. Denote the number of solutions that $j^{\text {th }}$ individual dominates:

$$
\mathbf{S}\left(X_{j}\right)=\left|\left\{X_{i} \mid X_{i} \in \mathbf{P}_{t}+\bar{P}_{t} \wedge X_{j}>X_{i}\right\}\right|
$$

where $X_{j}$ denotes the $i^{\text {th }}$ individual of the nondominated set A; $\mathbf{P}_{t}$ denotes the $t^{\text {th }}$ generation population; $\bar{P}_{t}$ denotes the $t^{\text {th }}$ generation archived population; $|\cdot|$ corresponds to the number of elements of a set; + represents the sum of two sets and the symbol; and $>$ indicates that the latter is dominated by the former.

(2) Raw Fitness $R\left(X_{j}\right)$. Denote the quality of other solutions that are better than the $i^{\text {th }}$ solution. Its lower value means better solution. In particular, when $R\left(X_{j}\right)$ is 0 , it means that there is no solution strictly better than this solution in the internal and external populations, and it is a noninferior solution. It is calculated as follows:

$$
R\left(X_{j}\right)=\sum_{X_{i} \in \mathbf{P}_{\mathbf{t}}+\bar{P}_{\mathbf{t}}, X_{j}>X_{i}} S\left(X_{i}\right)
$$

(3) Density $D\left(x_{i}\right)$. A number less than 1 indicates the density of the solution around the individual, and the larger the value is, the denser the solution set is. When the number of noninferior solutions exceeds the number of archive size $\bar{N}$, some of the solutions should be rejected in the dense area. However, the individuals in the sparser regions are retained to evolve in the next generation, so that the diversity of solutions can be maintained:

$$
\begin{aligned}
D\left(X_{j}\right) & =\frac{1}{\delta_{j}^{k}+2}, \\
k & =\sqrt{N+\bar{N}},
\end{aligned}
$$

where $\delta_{j}^{k}$ means the distance between $X_{j}$ individuals and the $k^{\text {th }}$ nearest individuals; $N$ denotes the population size; and $\bar{N}$ denotes the archive size.

(1) Actual fitness function $F\left(x_{i}\right)$ : it can be calculated as follows:

$$
F\left(x_{i}\right)=R\left(x_{i}\right)+D\left(x_{i}\right)
$$

3.2. Design of Grey Target Decision-Making with EWM. In this paper, grey target decision-making based on EWM is used to filter out the compromise solutions of the Pareto solution set. A bullseye is selected in grey target decisionmaking region formed by the solution set. The distance between each solution and the bullseye is taken as an important basis for grey target decision-making, and then the solutions are ranked according to the distance. Each solution of the solution set is considered as a separate decision solution. The weights and distances to the bullseye of each solution obtained by EWM do not rely on the evaluation and preference of experts, so that the decision is credible.
3.2.1. Design of the Effect Sample Matrix. The Pareto solution set $\mathbf{X}$ based on the SPEA2 algorithm is a matrix of $n$ rows and $m$ columns, which is also the set of unit outputs. Here, the absolute values of each solution in $\mathbf{X}$ can be taken as one of the decision indicators, as follows:

$$
X^{\prime}(i, j)=|X(i, j)|, \quad i=1,2, . ., n, j=1,2, \ldots, N .
$$

To consider the reduction of total power deviation and regulation mileage payment, two objective function values $F_{1}$ and $F_{2}$ are used as one of the evaluation indicators.

In this paper, we consider the limiting the variation of the output of each unit by adding an index $D$, representing the Euclidean distance of each solution of $\mathbf{X}^{\prime}$ to the origin, as follows:

$$
D_{i}=\sqrt{\sum_{j=1}^{m} X^{\prime}(i, j)^{2}} .
$$

Therefore, there are $m+3$ evaluation indicators, which are $m$ unit outputs, two objective function values, and Euclidean distance squared $D$. Therefore, the matrix of effect samples containing $n$ decision options and $m+3$ decision objectives is expressed as follows:

$$
\mathbf{X} \prime \prime=\left[\begin{array}{llll}
\mathbf{X} \prime & F_{1} & F_{2} & D
\end{array}\right] .
$$

3.2.2. Design of the Bullseye Vector. The operator $Z_{j}$ based on the principles of rewarding the best and punishing the worst is calculated as follows:

$$
Z_{j}=\frac{1}{n} \sum_{i=1}^{n} \mathbf{X} \prime(i, j), \quad j=1,2, \ldots, m+3 .
$$

Since the indicators are positive and the smaller they are, the better the program is, so this paper selects the cost-type indicator formula, the decision-making matrix $\mathbf{V}$ is calculated as follows:

$$
v_{i j}=\frac{z_{i j}-x_{i j}}{\max \left\{\max _{1 \leq i \leq n}\left\{x_{i j}\right\}-z_{j}, z_{j}-\min _{1 \leq i \leq n}\left\{x_{i j}\right\}\right\}} .
$$

Then, the decision matrix can be obtained $\mathbf{V}=\left(v_{i j}\right)$ $n \times(m+3) \cdot v_{j}^{0}=\max \left\{v_{j}^{i} \mid 1 \leq i \leq n\right\}, j=1,2, \ldots, m+3$ 。 Therefore, the selected bullseye vector is $v^{0}=\left\{v_{1}^{0}, v_{2}^{0}, \ldots, v_{m+3}^{0}\right\}$.

3.2.3. Design of Bullseye. In this paper, EWM is used to calculate the weights of the decision indicators, which is an objective method that does not depend on expert judgments or decision makers' preferences and helps to filter an objective solution from numerous solutions of the Pareto solution set and use this objective solution as the best compromise dispatch scheme. The weight $y_{i j}$ and entropy value $E_{j}$ are calculated based on the index values of each program, respectively, as follows: 


$$
\begin{aligned}
& y_{i j}=\frac{x_{i j}}{\sum_{i=1}^{n} x_{i j}}, \quad x_{i j} \geq 0, \\
& E_{j}=-\frac{1}{\ln n} \sum_{i=1}^{n} y_{i j} \ln y_{i j}, \quad E_{j}>0, \\
& \omega_{j}=\frac{\left(1-E_{j}\right)}{\sum_{j=1}^{m+3}\left(1-E_{j}\right)} .
\end{aligned}
$$

According to the bullseye vector $v^{0}=\left\{v_{1}^{0}, v_{2}^{0}, \ldots, v_{m+3}^{0}\right\}$ [13], the bullseye distances of each program can be expressed as follows:

$$
d_{i}=\left|v_{i}-v^{0}\right|=\mid \sqrt{\sum_{j=1}^{m+3} \omega_{j}\left(v_{i j}-v_{j}^{0}\right)^{2}} .
$$

The principle of the screening program is that the closer the indicator is to the bullseye, the better the solution.

3.2.4. Selection of Decision Options. When three consecutive AGC time intervals are taken, the set of three Pareto solution sets obtained by the SPEA2 algorithm is allowed to compare with the change in the decision solution, and the results are shown in Figure 3. It can be concluded from Figure 3 that the optimal rational point obtained by applying this decision is not necessarily in the geometric center of the set but is the result of combining the output of each unit as well as the biobjective value.

3.3. Calculation Flow. The whole calculation flow of solving BOCC by SPEA2 and grey target decision-making with EWM is provided in Table 2, where the termination condition of SPEA2 is set to the maximum number of iterations.

\section{Case Studies}

In this paper, the SPEA2 and grey target decision-making based BOCC are evaluated via the static test and dynamic test on the extended two-area LFC model by expanding the original single equivalent generating unit in area $\mathrm{A}$ into seven AGC units. The control time cycle of AGC is equal to $4 \mathrm{~s}$, and the price of regulation mileage is equal to $2 \$ / \mathrm{MW}$. The main parameters of transfer function parameters of the units are given in Table 3. And the main parameters of regulation of the units are given in Table 4 . The population size and the maximum number of iteration steps of all the algorithms are set to 50 and 50, respectively. And the archive size of SPEA2 is set to 50 .

4.1. Static Test Experiments. In the static test, two load disturbances are applied to the dynamic model, with $\Delta P_{D}=$ $50 \mathrm{MW}$ and $\Delta P_{D}=-50 \mathrm{MW}$. Here, 10 algorithms including the multiobjective immune algorithm with nondominated neighbor-based selection (NNIA) [22], NSGA-II, strength Pareto evolutionary algorithm based on reference direction for multiobjective (SPEAR) [23], SPEA2, a decision variable clustering-based evolutionary algorithm for large-scale many-objective (LMEA) [24], a cellular genetic algorithm for multiobjective optimization (MOCELL) [25], a proposal for multiple-objective particle swarm optimization (MOPSO) [26], an evolutionary many-objective optimization algorithm using reference point-based nondominated (NSGA-III) [27], many-objective evolutionary optimization based on reference points (PREA) [28], and region-based selection in evolutionary multiobjective optimization (PESAII) [29] are selected for comparison.

Figure 4 provides the comparison of the local Pareto front of the ten algorithms. Figure 4(a) shows that the solutions obtained by LMEA and PESAII deviate obviously from the ideal Pareto front, in which the Pareto front obtained by SPEA2 tends to be better than the fronts of NSGA-II and NSGA-III. Figure 4(b) shows that the solutions obtained by LMEA, SPEAR, and PESAII deviate obviously from the ideal Pareto front, while the Pareto front obtained by SPEA2 tends to be better than the fronts of NSGA-II, MOCELL, and NSGA-III. Meanwhile, the Pareto solutions of SPEA2 are evenly and widely distributed on the local Pareto front.

Figure 4(c) shows that Pareto fronts obtained by 10 algorithms with 10 runs are converted into an approximate ideal Pareto surface. To better compare the convergence and diversity of the algorithms, the performance indexes of four algorithms are evaluated in Table 5, including inverted generational distance (IGD) [30], generational distance (GD) [31], pure diversity (PD) [32], hyper volume (HV) [33], DM (diversity metric) [34], spread [35], and spacing [36], where IGD [37] and HV denote the accuracy and diversity of algorithm; GD considers the accuracy of the algorithm; PD and spread denote the diversity of the algorithm; spacing denotes the evenness of the solutions obtained by algorithm; GD, IGD, spread, and spacing are the negative indexes, while DM, $\mathrm{HV}$, and PD are the positive indexes [38-40].

It is evident that the SPEA2 outperforms the other three algorithms in the extend two-area LFC model at the two disturbances:

(1) The SPEA2 has the smallest GD value among the average data of the algorithm metrics, representing that it has a good merit of convergence performance.

(2) It has the largest DM and HV average, which demonstrates that SPEA2 has the largest objective space covered by the Pareto front.

(3) It has the smallest spread and spacing average, i.e., which indicates that the Pareto solutions obtained by SPEA2 are evenly and widely distributed on the Pareto front.

(4) It also has the smallest running average time and satisfies the requirement that the time interval of 


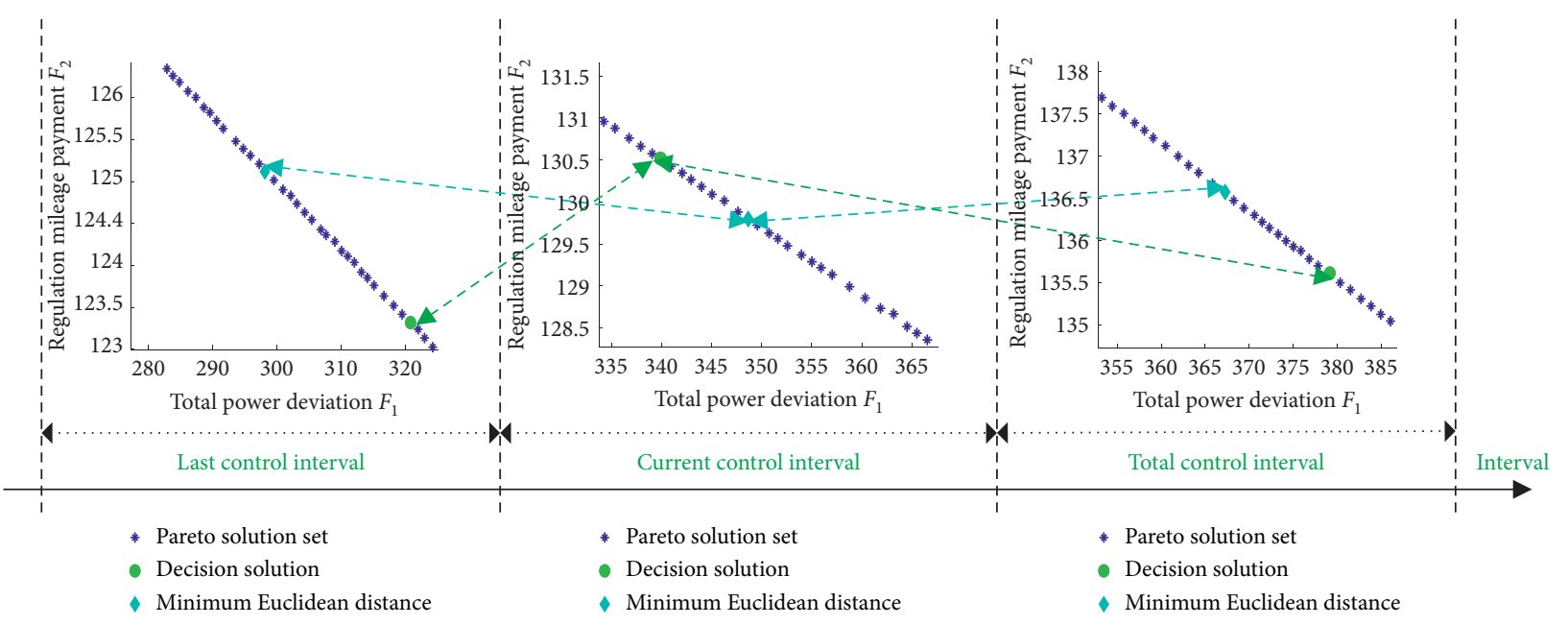

FIgURE 3: The selection of decision options.

TABLE 2: The execution procedure of SPEA2 for BOCC.

Input: The total power regulation command for the current control interval.

1: Initialize the parameters of SPEA2;

2: Initialize the parent population and archive of SPEA2;

3: Calculate the initial dispatch solution by equations (1)-(7);

4: Compute the objective function for each individual by equations (8)-(10);

5: Evaluate the fitness function for each individual by equation (11);

6: Merge the generation population and the generation archived population into one.

7: Calculate the parameters of the new set by equations (12)-(16);

8: Compare the number of noninferior solutions and archive size, and then perform the truncation or reproduction to fill the archive set.

9: Generate the offspring population by applying recombination and mutation operators to the set

10: If the termination condition of SPEA2 is satisfied, then go to the next step; otherwise, return to Step 4;

11: Find the best compromise solution from multiple solutions in Pareto solution set by grey target decision-making theory in equation (17)-(25).

Output: The best power regulation inputs for all AGC units.

TABLE 3: Transfer function parameters of AGC units.

\begin{tabular}{lc}
\hline Generation type & Parameters $(\mathrm{s})$ \\
\hline Coal-fired & $T 2=5, T 3=0.08, T 4=10, T 5=0.3$ \\
LNG & $T 2=2, T 3=0.05, T 4=5, T 5=0.2$ \\
Hydro & $T 6=1, T 7=5, T 8=0.513$ \\
WT, PV & $T 1=0.01$ \\
ESRs & $T 1=2$ \\
\hline
\end{tabular}

AGC dispatch is less than $4 \mathrm{~s}$, which means that SPEA2 can converge to the Pareto front much faster than other three algorithms.

\subsection{Dynamic Test}

4.2.1. Step Load Disturbance. To better verify the superiority of SPEA2 and grey target decision-making, the following dynamic simulation tests are performed in real-time optimization of BOCC. This paper introduces a comparison using the adjustable capacity method, called the proportional (PROP) method [41]. PROP is an engineering calculation method, which distribute the dispatching signal of the unit by using the proportion of the adjustable capacity of the unit. Therefore, the output of the $i^{\text {th }}$ AGC unit at $k^{\text {th }}$ time interval is calculated as follows:

$$
\Delta P_{i}^{\text {out }}(k)= \begin{cases}\Delta P_{c}(k) \cdot \frac{\Delta P_{i}^{\max }}{\sum_{i=1}^{n} \Delta P_{i}^{\max },} & \text { if } \Delta P_{c}(k) \geq 0, \\ \Delta P_{c}(k) \cdot \frac{\Delta P_{i}^{\min }}{\sum_{i=1}^{n} \Delta P_{i}^{\min },} & \text { if } \Delta P_{c}(k)<0 .\end{cases}
$$

Then, two load disturbances (see Figures 5 and 6) are applied to the extended two-area LFC model, which are $\Delta P_{D}=70 \mathrm{MW}$ and $\Delta P_{D}=-50 \mathrm{MW}$.

Figure 5(a) reveals that the proposed SPEA2 with grey target decision-making and EWM can get a more minor error between the total input power and total output power than PROP via the regulation coordination between ESRs and other units, as shown in Figure 5(b). Hence, it slightly reduces the peak of frequency deviation due to it can match the total regulation command closely. Moreover, it results a lower regulation mileage payment than PROP, as illustrated in Figure 7(a).

Figure 6(a) shows that compared to the PROP, the proposed method obtains a less power deviation, reduces the overshoot of the total power command, and makes the total 
TABLE 4: Main parameters of AGC units in area A of the two-area LFC model.

\begin{tabular}{|c|c|c|c|c|c|}
\hline Unit no. & Type & $\mathrm{Td}(\mathrm{s})$ & $\Delta P^{\text {rate }}(\mathrm{MW} / \mathrm{min})$ & $\Delta P_{\max }(\mathrm{MW})$ & $\Delta P_{\min }(\mathrm{MW})$ \\
\hline$G_{1}$ & Coal-fired & 60 & 30 & 50 & -50 \\
\hline$G_{2}$ & LNG & 20 & 18 & 30 & -30 \\
\hline$G_{3}$ & Hydro & 5 & 150 & 20 & -10 \\
\hline$G_{4}$ & WT & 1 & - & 15 & -5 \\
\hline$G_{5}$ & PV & 1 & - & 10 & -10 \\
\hline$G_{6}$ & ESRs1 & 1 & - & 8 & -12 \\
\hline$G_{7}$ & ESRs2 & 1 & - & 7 & -13 \\
\hline
\end{tabular}
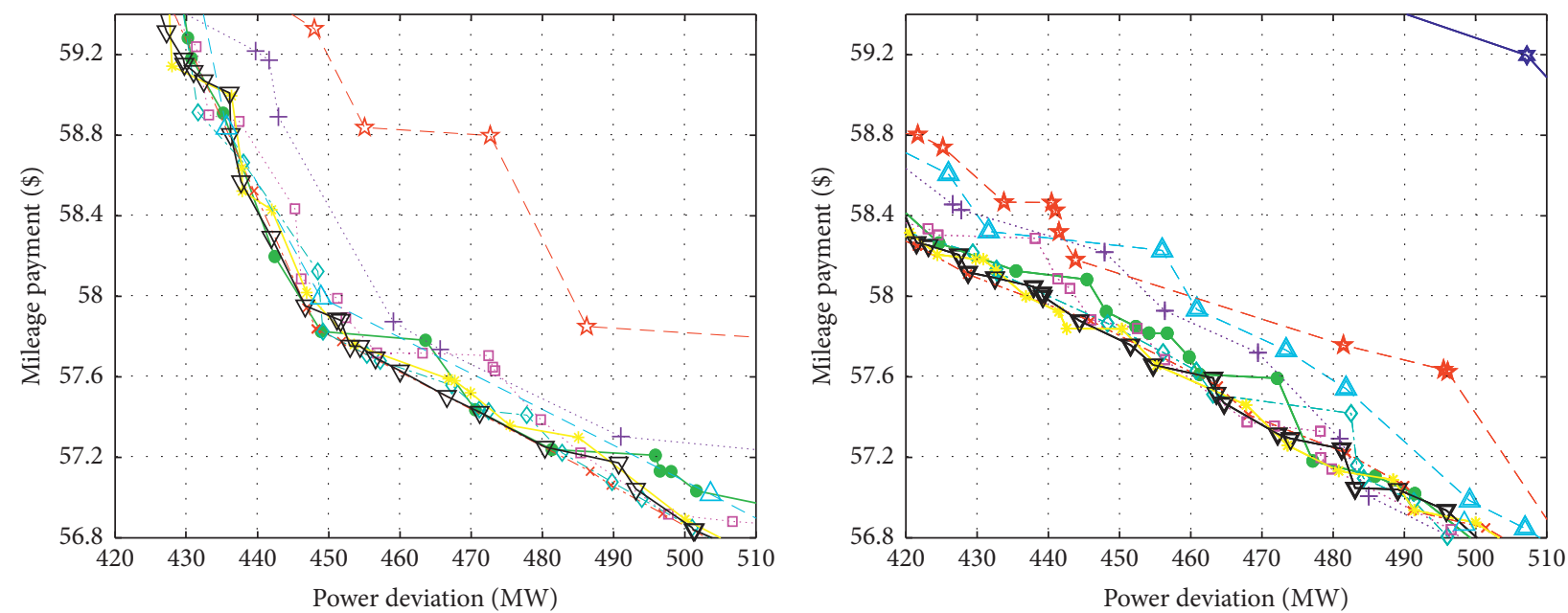

\begin{tabular}{|c|c|c|}
\hline LMEA & … NSGAII & $\nabla$ RFMO \\
\hline$\longrightarrow$ MOCELL & $\cdot \cdot+\cdot \cdot$ NSGAIII & $-A-$ SPEAR \\
\hline $\begin{array}{l}-x-\text { MOPSO } \\
-\diamond-\text { NNIA }\end{array}$ & * PREA & -资- PESAI \\
\hline
\end{tabular}

(a)

\begin{tabular}{|c|c|c|}
\hline LMEA & … NSGAII & $\nabla$ RFMO \\
\hline - MOCELL & $\cdot \cdot+\cdot$ NSGAIII & $-A-$ SPEAR \\
\hline$-x-$ MOPSO & * PREA & -支- PESAII \\
\hline
\end{tabular}

$\diamond-$ NNIA

(b)

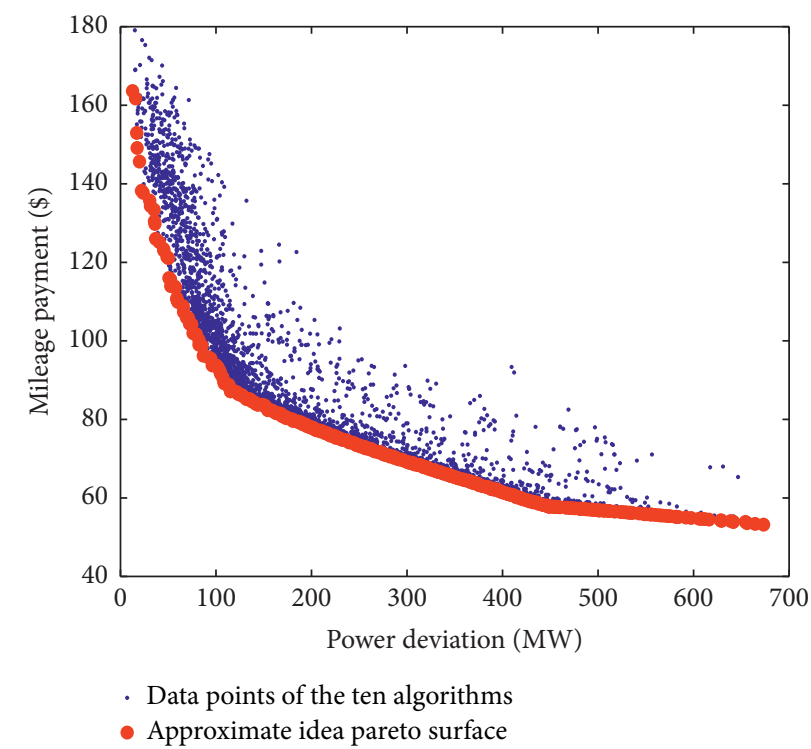

(c)

Figure 4: Comparison of the Pareto front surface obtained by 10 algorithms on the extended two-area LFC model: $(\mathrm{a}) \Delta P_{D}=70 \mathrm{MW}$, (b) $\Delta P_{D}=70 \mathrm{MW}$, and (c) select the approximate ideal Pareto surface. 
TABLE 5: Comparison of performance metrics of algorithms.

\begin{tabular}{|c|c|c|c|c|c|c|c|c|c|c|}
\hline$\Delta P_{D}$ & Func & & IGD & GD & PD & $\mathrm{HV}$ & $\mathrm{DM}$ & Spread & Spacing & $T(\mathrm{~s})$ \\
\hline \multirow{8}{*}{$50 \mathrm{~W}$} & \multirow{2}{*}{ NNIA } & Ave & 8.50 & 1.01 & $3.36 E+05$ & 0.572 & 0.726 & 0.663 & 6.04 & $7.33 E-02$ \\
\hline & & Std & 2.96 & 0.29 & $7.18 E+04$ & 0.004 & 0.059 & 0.085 & 1.06 & $1.34 E-03$ \\
\hline & \multirow{2}{*}{ NSGAII } & Ave & 11.23 & 0.94 & $2.99 E+05$ & 0.571 & 0.684 & 0.696 & 4.96 & $6.50 E-02$ \\
\hline & & Std & 5.16 & 0.26 & $5.21 E+04$ & 0.008 & 0.047 & 0.064 & 0.69 & $2.58 E-03$ \\
\hline & \multirow{2}{*}{ SPAR2 } & Ave & 11.08 & 0.64 & $2.85 E+05$ & 0.573 & 0.752 & 0.475 & 3.62 & $6.49 E-02$ \\
\hline & & Std & 5.33 & 0.17 & $7.60 E+04$ & 0.007 & 0.076 & 0.090 & 0.79 & $1.92 E-03$ \\
\hline & \multirow{2}{*}{ SPEAR } & Ave & 12.20 & 0.98 & $2.47 E+05$ & 0.562 & 0.634 & 0.826 & 9.01 & $6.63 E-02$ \\
\hline & & Std & 4.17 & 0.34 & $3.47 E+04$ & 0.006 & 0.057 & 0.076 & 2.06 & $2.98 E-03$ \\
\hline \multirow{8}{*}{$-50 \mathrm{~W}$} & \multirow{2}{*}{ NNIA } & Ave & 12.54 & 0.66 & $2.18 E+05$ & 0.485 & 0.689 & 0.735 & 5.44 & $7.79 E-02$ \\
\hline & & Std & 11.87 & 0.15 & $2.47 E+04$ & 0.003 & 0.065 & 0.070 & 1.23 & $5.13 E-03$ \\
\hline & \multirow{2}{*}{ NSGAII } & Ave & 14.68 & 0.37 & $2.15 E+05$ & 0.485 & 0.663 & 0.759 & 4.73 & $6.48 E-02$ \\
\hline & & Std & 11.37 & 0.14 & $3.38 E+04$ & 0.007 & 0.079 & 0.056 & 1.05 & $2.87 E-03$ \\
\hline & \multirow{2}{*}{ SPAR2 } & Ave & 22.35 & 0.45 & $1.74 E+05$ & 0.479 & 0.692 & 0.523 & 2.96 & $6.47 E-02$ \\
\hline & & Std & 25.49 & 0.11 & $3.52 E+04$ & 0.009 & 0.151 & 0.143 & 0.87 & $2.46 E-03$ \\
\hline & \multirow{2}{*}{ SPEAR } & Ave & 13.12 & 0.61 & $2.15 E+05$ & 0.487 & 0.612 & 0.879 & 7.76 & $6.70 E-02$ \\
\hline & & Std & 6.04 & 0.15 & $4.79 E+04$ & 0.004 & 0.062 & 0.067 & 2.30 & $2.84 E-03$ \\
\hline
\end{tabular}

power output curve markedly closer to the total command curve. However, it results a higher regulation mileage payment (see Figure 7(a)), because it assigns larger commands to the AGC units with fast performance scores.

4.2.2. Continuous Step Load Disturbance. In the following dynamic simulation test, two continuous step load disturbances with 120 control intervals a step and 300 control intervals a step (see Figures 8(b) and 9(c)) are applied to the extended two-area LFC mode.

At the short-time interval step, Figure 8(a) illustrates that the proposed method can also gain a slightly smaller power deviation. After the 460 control interval (see Figure 8(a)), the mileage payment of PROP quickly exceeds that of SPEA2. After reaching the lower limit capacity, the outputs of the PV unit and wind power remain almost static (see Figure 8(b)). However, for PROP, those outputs continue to change dramatically during the simulation.

At the long-time interval step, Figure 9(a) shows that the proposed method also gets a smaller power deviation and a smaller frequency deviation. Moreover, it makes a lower regulation mileage payment (see Figure 7(a)) because PROP distributes more input power command to the PV unit and wind power, while the disturbance borne by wind power and PV unit is shared by ESR units after SPEA2 optimization. It can be concluded that at the short-time interval step, the proposed method can help significantly reduce the mileage payment for IOSs. On the contrary, at the long-time interval step, it can make the total output command well close to the total input command.

To further discuss the relationship between the regulation mileage payment and regulated output power of new energy units and ESRs, Figure 7(a) displays the payment changes under the four disturbance cases. In case of ideal single-step disturbance, due to the stochastic character of the calculation results of the multiobjective algorithm, SPEA2 has uncertainty in reducing the regulation mileage payment, but in the continuous step disturbance case, the algorithm plays a significant role in reducing the regulation mileage payment. In case of successive step disturbance, the two curves before and after the optimization have an intersection point (see Figure $7(\mathrm{a})$ ) due to the changes of the total command assignment scheme (see Figures 7(b) and 7(c)). In the starting phase of the disturbance, because the proposed method assigns mainly units with fast performance scores and high regulation price to participate in the AGC dispatch, it can obtain a slightly higher regulation mileage payment than PROP. However, in the middle stage of disturbance, the PROP still involves wind power and PV unit in regulation frequency, and the regulation capacity of ESR units is not fully utilized. However, in the dispatch with SPEA2 participation, the disturbance borne by wind power and PV units is shared by ESR units, and the superior regulation performance of ESR units is still maximized, so the dispatching payment with SPEA2 participation will be lower than the payment before optimization. In conclusion, ESRs play an important role in AGC dispatch, and the proposed method can make full use of this advantage.

To further test the performance of the SPEA2 algorithm, this example compares the online optimization results of the four cases separately, as shown in Table 6, where accuracy is used to measure the approximate degree of the actual regulation output and the regulation command curve during the simulation time. It can be found two main points from Table 5 compared with PROP, as follows:

(1) The proposed method can significantly improve the power quality for power grid by reducing the power deviation and improving each performance index of the system dynamic response, especially in the case of continuous short-time interval step disturbances. The power deviation, average $|\Delta f|$, and $|\mathrm{ACE}|$ reduce $66.8 \%, 8.0 \%$, and $5.2 \%$, respectively, in the case of continuous and long-time interval step load disturbance. The power deviation, average $|\Delta f|$, and $\mid$ 


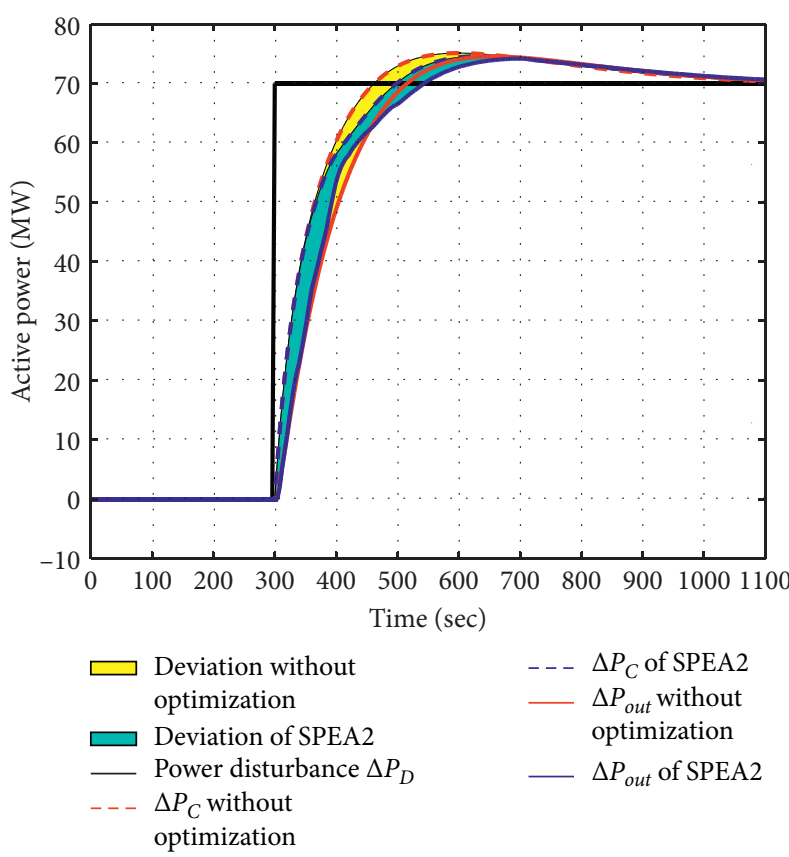

(a)

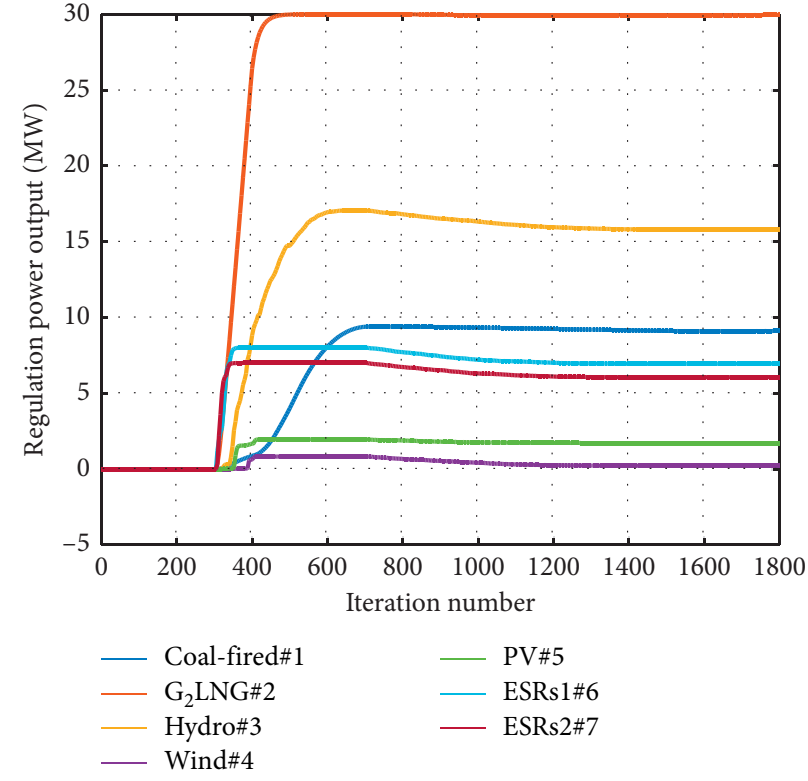

(b)

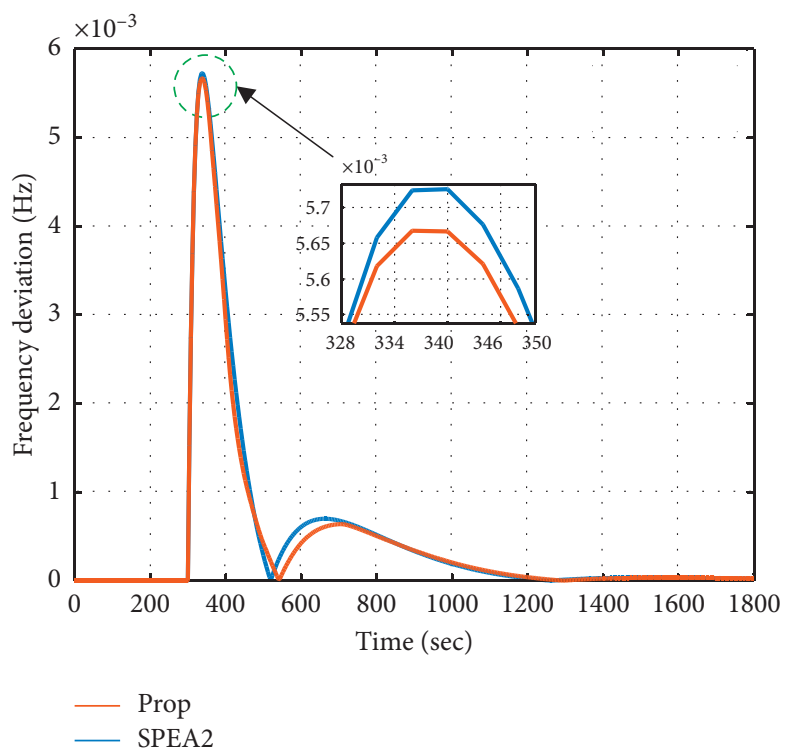

(c)

FIGURE 5: Real-time optimization results obtained on the extended two-area LFC model when $\Delta P_{D}=70 \mathrm{MW}$, (a) overall power deviation, (b) regulation power output obtained by SPEA2 with grey target decision-making, and (c) frequency deviation.

ACE $\mid$ reduce $45.9 \%, 5.9 \%$, and $6.4 \%$, respectively, in the case of continuous and short-time interval step load disturbance.
(2) The proposed method can obviously reduce the regulation mileage payment and thus rise the economy operating for ISOs, especially in the case of 

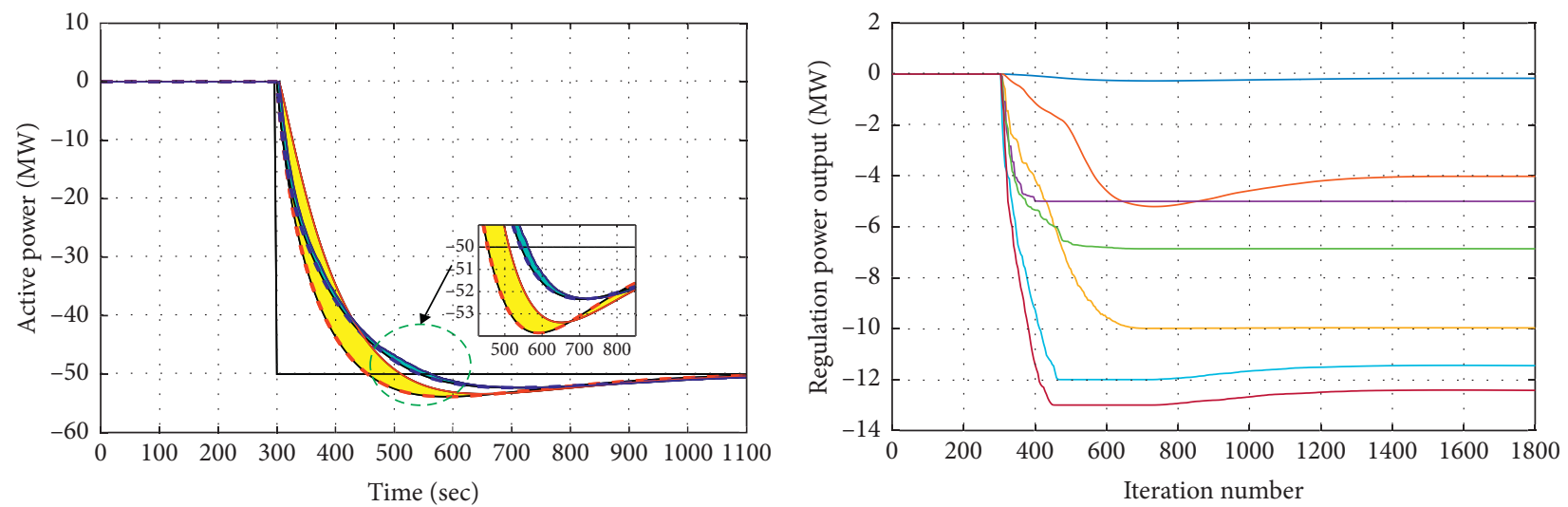

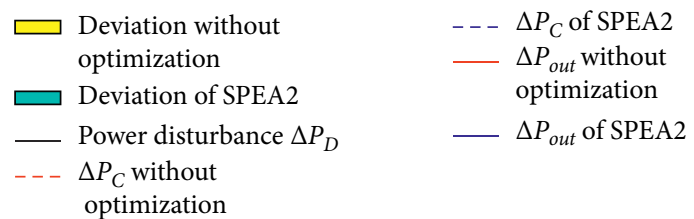

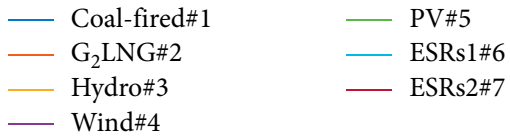

(a)

(b)

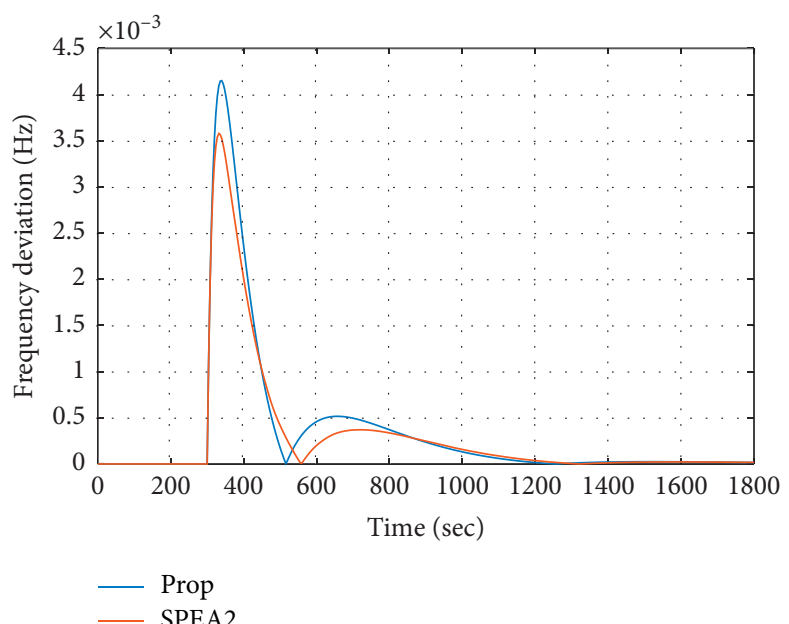

(c)

FIGURE 6: Real-time optimization results obtained on the extended two-area LFC model when $\Delta P_{D}=-50 \mathrm{MW}$, (a) overall power deviation, (b) regulation power output obtained by SPEA2 with grey target decision-making, and (c) frequency deviation.
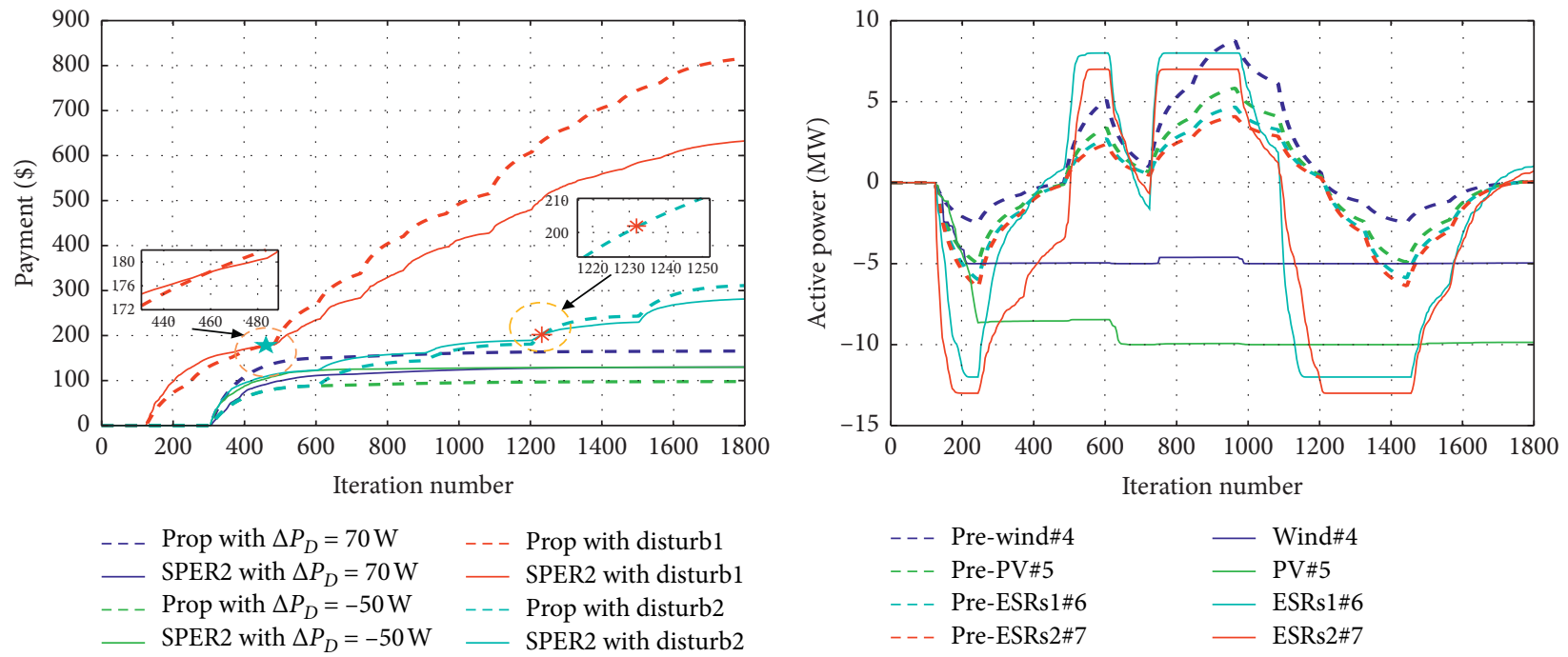

(a)

(b)

Figure 7: Continued. 


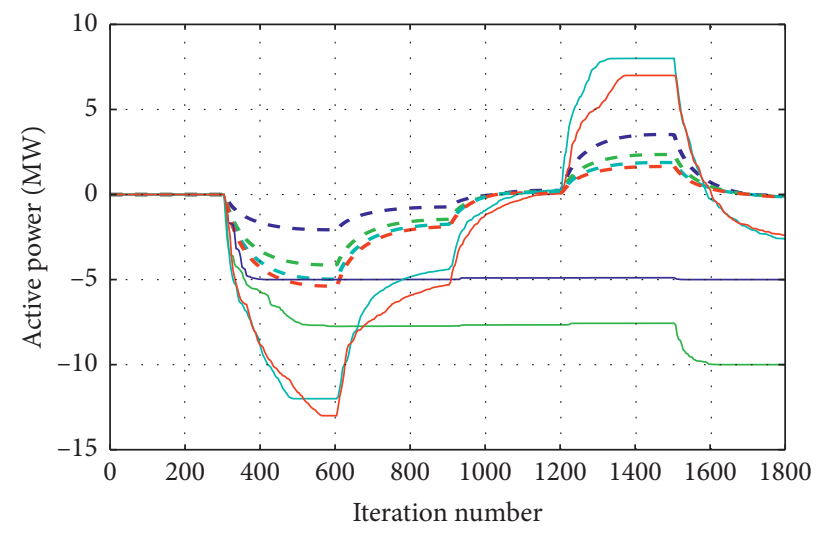

$$
\begin{array}{ll}
\text { - - - Pre-wind\#4 } & - \text { Wind\#4 } \\
\text { - - - Pre-PV\#5 } & - \text { PV\#5 } \\
\text { - - - Pre-ESRs1\#6 } & - \text { ESRs1\#6 } \\
\text { - - - Pre-ESRs2\#7 } & - \text { ESRs2\#7 }
\end{array}
$$

(c)

Figure 7: (a) The payment under four disturbance cases. Comparison of regulated output power of new energy units and ESRs before and after optimization: (b) under a continuous and short-time interval step load disturbance and (c) under a continuous and long-time interval step load disturbance.

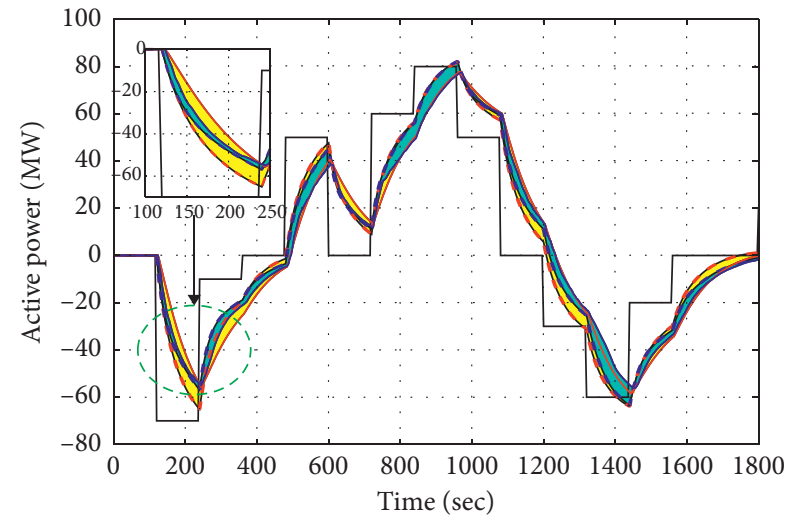

$\sqsubset$ Deviation without optimization

$\square$ Deviation of SPEA2

- Power disturbance $\Delta P_{D}$

- - $\Delta P_{C}$ without optimization

$$
\begin{array}{ll}
-- & \Delta P_{C} \text { of SPEA2 } \\
- & \Delta P_{\text {out }} \text { without } \\
& \text { optimization } \\
- & \Delta P_{\text {out }} \text { of SPEA2 }
\end{array}
$$

(a)

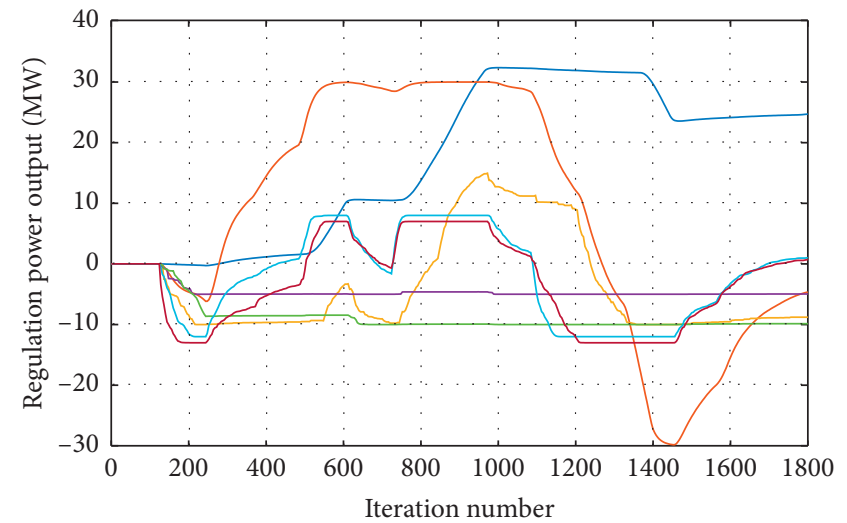

- PV\#5

— ESRs1\#6

— ESRs2\#7

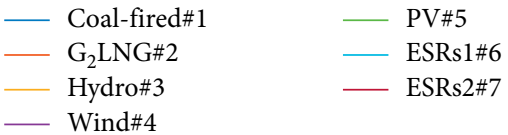

(b)

Figure 8: Continued. 


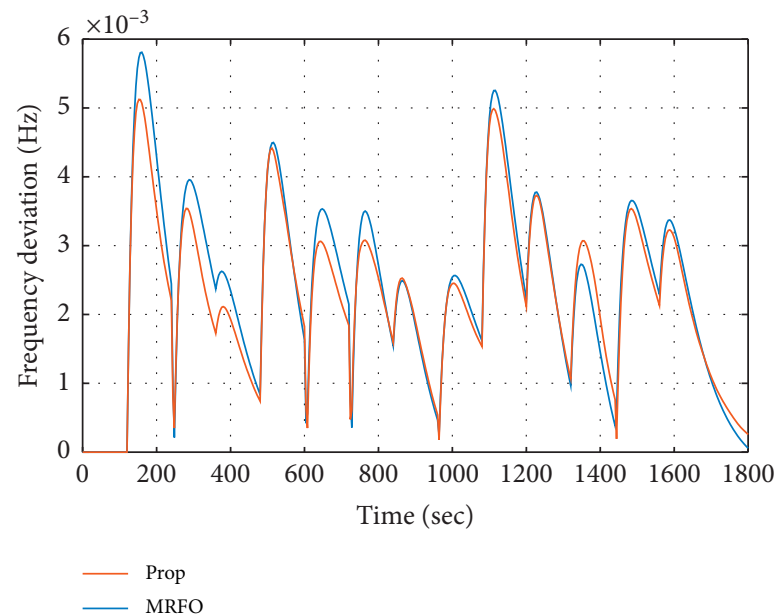

(c)

FIGURE 8: Online optimization results obtained by SPEA2 under a continuous and short-time interval step load disturbance in the extended two-area LFC model, (a) overall power deviation, (b) regulation power output obtained by SPEA2 with grey target decision-making, and (c) frequency deviation.

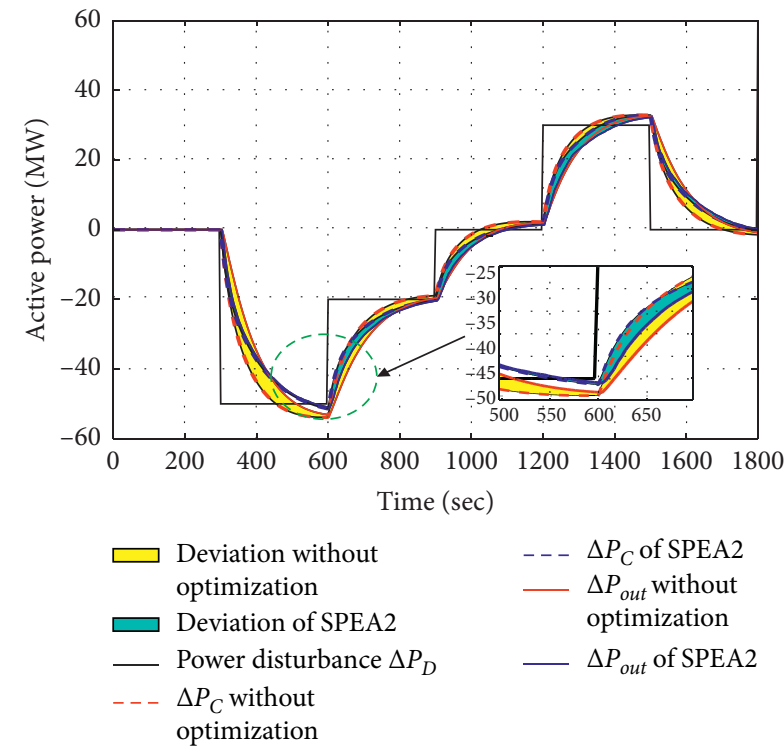

(a)
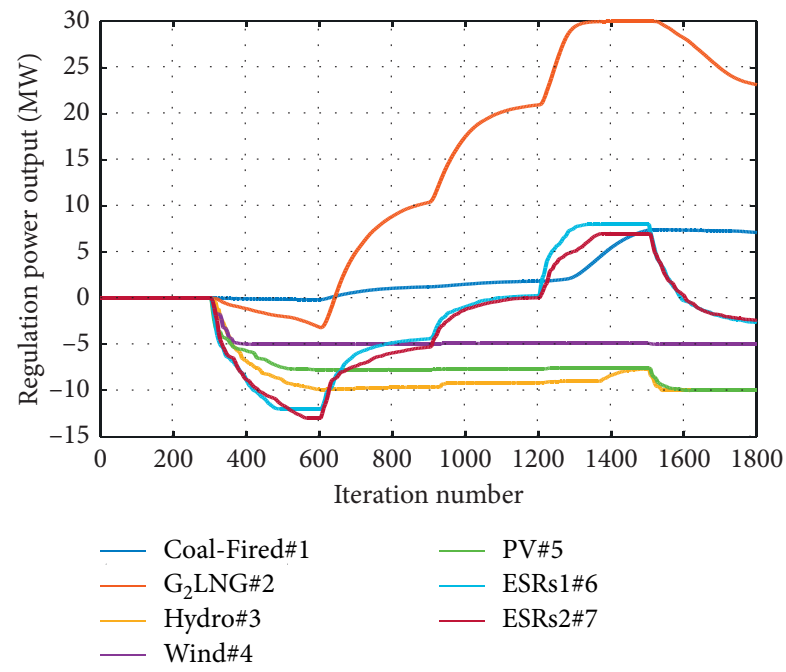

(b)

Figure 9: Continued. 


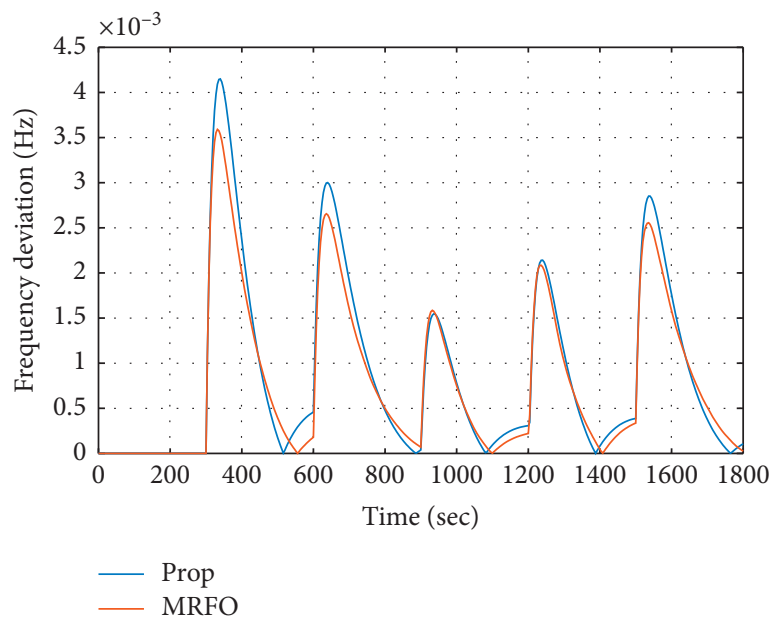

(c)

FIGURE 9: Online optimization results obtained by SPEA2 under a continuous and long-time interval step load disturbance in the extended two-area LFC model, (a) overall power deviation, (b) regulation power output obtained by SPEA2 with grey target decision-making, and (c) frequency deviation.

TABLE 6: Result comparison of online optimization under different disturbances.

\begin{tabular}{|c|c|c|c|c|c|c|c|c|c|c|}
\hline \multirow[t]{2}{*}{$\Delta P_{D}$} & \multirow[t]{2}{*}{ Method } & \multicolumn{2}{|c|}{$\begin{array}{l}|\mathrm{ACE}| \\
(\mathrm{MW})\end{array}$} & \multicolumn{2}{|c|}{$|\Delta f|(\mathrm{Hz})$} & \multicolumn{2}{|c|}{ CPS1 (\%) } & \multirow[t]{2}{*}{ Deviation (MW) } & \multirow[t]{2}{*}{ Accuracy (\%) } & \multirow[t]{2}{*}{ Payment (\$) } \\
\hline & & Avg & $\operatorname{Max}$ & Avg & Max & Avg & Min & & & \\
\hline \multirow{2}{*}{$70 \mathrm{MW}$} & PROP & 0.89 & 11.17 & $4.78 E-04$ & $5.73 E-03$ & 199.99 & 199.82 & 550.06 & 81.34 & 165.58 \\
\hline & SPEA2 & 0.88 & 11.06 & $4.52 E-04$ & $5.67 E-03$ & 199.99 & 199.82 & 383.36 & 81.87 & 130.09 \\
\hline \multirow{2}{*}{$-50 \mathrm{MW}$} & PROP & 0.64 & 8.09 & $3.46 E-04$ & $4.15 E-03$ & 199.99 & 199.90 & 426.14 & 81.19 & 97.87 \\
\hline & SPEA2 & 0.61 & 6.93 & $3.04 E-04$ & $3.58 E-03$ & 199.99 & 199.93 & 53.36 & 82.87 & 126.95 \\
\hline \multirow{2}{*}{ Disturb 1 (see Figure 8(a)) } & PROP & 4.72 & 11.33 & $2.38 E-03$ & $5.81 E-03$ & 199.96 & 199.81 & 2854.38 & 74.23 & 816.35 \\
\hline & SPEA2 & 4.42 & 9.95 & $2.24 E-03$ & $5.12 E-03$ & 199.96 & 199.85 & 1544.57 & 82.73 & 632.56 \\
\hline \multirow{2}{*}{ Disturb 2 (see Figure 9(a)) } & PROP & 1.92 & 8.09 & $8.71 E-04$ & $4.15 E-03$ & 199.99 & 199.90 & 1240.69 & 70.31 & 311.12 \\
\hline & SPEA2 & 1.82 & 6.95 & $8.01 E-04$ & $3.59 E-03$ & 199.99 & 199.93 & 411.56 & 78.75 & 281.25 \\
\hline
\end{tabular}

continuous long-time interval step disturbances. Particularly, in the two continuous disturbance test, the regulation mileage payments reduce by $22.5 \%$ and $9.6 \%$.

\section{Conclusions}

In summary, the presented work includes the following three contributions:

(1) The proposed BOCC can effectively coordinate various frequency regulation resources and ESRs for AGC, which can simultaneously reduce the total power deviation and the regulation mileage payment.

(2) The presented SPEA2 can converge to a high-quality Pareto front for BOCC in the relatively short time, while grey target decision-making with EWM can effectively select a compromise dispatch scheme that makes full use of the advantage of ESRs in frequency regulation to consider both of the operating economy and power quality.
(3) The simulation results of the extended two-area LFC model verify that the combination of SPEA2 and grey target decision-making with EWM can effectively solve BOCC. It can effectively improve the response performance by reducing the $|\mathrm{ACE}|$, average $|\Delta f|$, and total power deviation and simultaneously rise the operating economy for IOSs by reducing the total regulation mileage payment.

To further improve the operating economy and power quality, the future studies will focus on the higher-participated renewable energy and smaller control periodic. Furthermore, the method based on model predictive control or deep learning will be the key technology to solve BOCC.

\section{Data Availability}

The data that support the findings of this study are available on request from the corresponding author. The data are not publicly available due to privacy or ethical restrictions. 


\section{Conflicts of Interest}

The authors declare that they have no conflicts of interest.

\section{Acknowledgments}

This work was jointly supported by Research on Regional Power Grid Frequency Regulation with Renewable Energy Participation (YNKJXM20191240), the Project of the Scientific Research Staring Foundation of Shantou University (no. NTF20009), and the Open Project of Key Laboratory of Computational Intelligence and Signal Processing for Ministry of Education (no. 2019A002).

\section{References}

[1] L. Xi, J. Chen, Y. Huang et al., "Smart generation control based on multi-agent reinforcement learning with the idea of the time tunnel," Energy, vol. 153, pp. 977-987, 2018.

[2] V. Lakshmanan, M. Marinelli, J. Hu, and H. W. Bindner, "Provision of secondary frequency control via demand response activation on thermostatically controlled loads: solutions and experiences from Denmark," Applied Energy, vol. 173, pp. 470-480, 2016.

[3] Z. Liu, H. Zhang, S. Gao, X. Ma, and Y. Lu, "The world's largest all-vanadium redox flow battery energy storage system for a wind farm," Energy Storage Science and Technology, vol. 3, no. 1, pp. 71-77, 2014.

[4] D. M. Davies, M. G. Verde, O. Mnyshenko et al., "Combined economic and technological evaluation of battery energy storage for grid applications," Nature Energy, vol. 4, no. 1, pp. 42-50, 2019.

[5] B. Xu, Y. Dvorkin, D. S. Kirschen, C. A. Silva-Monroy, and J. P. Watson, "A comparison of policies on the participation of storage in U.S. frequency regulation markets," in Proceedings of the 2016 IEEE Power and Energy Society General Meeting (PESGM), pp. 1-5, Boston, MA, USA, July 2016.

[6] U. S. Federal Energy Regulatory Commission, FERC 755, Dockets RM11-7-000 AD10-11-000, U. S. Federal Energy Regulatory Commission, Washington, DC, USA, 2011.

[7] A. D. Papalexopoulos and P. E. Andrianesis, "Performancebased pricing of frequency regulation in electricity markets," IEEE Transactions on Power Systems, vol. 29, no. 1, pp. 441449, 2014.

[8] Federal Energy Regulatory Commission, Electric storage Participation In Markets Operated by Regional Transmission Organizations and Independent System Operators, Order No. 841, 162 FERC g 61, p. 127, Federal Energy Regulatory Commission, Washington, DC, USA, 2018.

[9] X. Zhang, T. Tan, T. Yu, B. Yang, and X. Huang, "Bi-objective optimization of real-time AGC dispatch in a performancebased frequency regulation market," CSEE Journal of Power and Energy Systems, 2020.

[10] D. Gong, B. Xu, Y. Zhang, Y. Guo, and S. Yang, "A similaritybased cooperative Co-evolutionary algorithm for dynamic interval multiobjective optimization problems," IEEE Transactions on Evolutionary Computation, vol. 24, no. 1, pp. 142-156, 2020.

[11] Y. Tian, C. Lu, X. Zhang, K. Tan, and Y. Jin, "Solving largescale multi-objective optimization problems with sparse optimal solutions via unsupervised neural networks," IEEE Transactions on Cybernetics, 2020.
[12] C. Wang, R. Xu, J. Qiu, and X. Zhang, “AdaBoost-inspired multi-operator ensemble strategy for multi-objective evolutionary algorithms," Neurocomputing, vol. 384, pp. 243-255, 2020.

[13] F. Cheng, Q. Zhang, Y. Tian, and X. Zhang, "Maximizing receiver operating characteristics convex hull via dynamic reference point-based multi-objective evolutionary algorithm," Applied Soft Computing, vol. 86, pp. 1-11, 2020.

[14] L. Zhang, J. Xia, F. Cheng, J. Qiu, and X. Zhang, "Multiobjective optimization of critical node detection based on cascade model in complex networks," IEEE Transactions on Network Science and Engineering, vol. 7, no. 3, pp. 2052-2066, 2020.

[15] F. Cheng, J. Chen, J. Qiu, and L. Zhang, “A subregion division based multi-objective evolutionary algorithm for SVM training set selection," Neurocomputing, vol. 394, pp. 70-83, 2020.

[16] E. Zitzler, M. Laumanns, and L. Thiele, "SPEA2: improving the strength pareto evolutionary algorithm," TIK-Report, vol 103, Eidgenössische Technische Hochschule Zürich, Zürich, Switzerland, 2001.

[17] K. Deb, A. Pratap, S. Agarwal, and T. Meyarivan, "A fast and elitist multiobjective genetic algorithm: NSGA-II," IEEE Transactions on Evolutionary Computation, vol. 6, no. 2, pp. 182-197, 2002.

[18] Z. Gu, D. Niu, and H. Wang, "Multiple attribute grey target decision making model based on linear combination weights determining and entropy," International Journal of Business and Management, vol. 3, no. 1, pp. 106-111, 2008.

[19] J. W. Ra, "Hierarchical decision process," technology management: the new international language," in Proceedings of the-PICMET '91, pp. 595-599, Portland, OR, USA, October 1991.

[20] D. Norman and H. Olaf, "An experimental application of the Delphi method to the use of experts," Management Science, vol. 9, no. 3, pp. 458-467, 1963.

[21] T. Yu, Y. M. Wang, W. J. Ye, B. Zhou, and K. W. Chan, "Stochastic optimal generation command dispatch based on improved hierarchical reinforcement learning approach," IET Generation, Transmission \& Distribution, vol. 5, no. 8, pp. 789-797, 2011.

[22] M. Gong, L. Jiao, H. Du, and L. Bo, "Multi-objective immune algorithm with nondominated neighbor-based selection," Evolutionary Computation, vol. 16, no. 2, 2008.

[23] S. Jiang and S. Yang, "A strength Pareto evolutionary algorithm based on reference direction for multiobjective and many-objective optimization," IEEE Transactions on Evolutionary Computation, vol. 21, no. 3, pp. 329-346, 2017.

[24] X. Zhang, Y. Tian, R. Cheng, and Y. Jin, "A decision variable clustering-based evolutionary algorithm for large-scale manyobjective optimization," IEEE Transactions on Evolutionary Computation, vol. 22, no. 1, pp. 97-112, 2016.

[25] H. Kor, H. Iranmanesh, H. Haleh, and S. M. Hatefi, “A multiobjective genetic algorithm for optimization of cellular manufacturing system," in Proceedings of the 2009 International Conference on Computer Engineering and Technology, pp. 252-256, Singapore, January 2009.

[26] C. C. Coello and M. S. Lechuga, "MOPSO: a proposal for multiple objective particle swarm optimization," in Proceedings of the 2002 IEEE Congress on Evolutionary Computation, pp. 1051-1056, Honolulu, HI, USA, May 2002.

[27] K. Deb and H. Jain, "An evolutionary many-objective optimization algorithm using reference-point-based nondominated sorting approach, part I: solving problems with 
box constraints," IEEE Transactions on Evolutionary Computation, vol. 18, no. 4, pp. 577-601, 2014.

[28] Y. Liu, D. Gong, X. Sun, and Y. Zhang, "Many-objective evolutionary optimization based on reference points," Applied Soft Computing, vol. 50, pp. 344-355, 2017.

[29] D. W. Corne, N. R. Jerram, J. D. Knowles, and M. J. Oates, "PESA.-II: region-based selection in evolutionary multi-objective optimization," in Proceedings of the 3rd Annual Conference on Genetic and Evolutionary Computation, pp. 283-290, San Francisco, CA, USA, January 2001.

[30] A. Zhou, Y. Jin, Q. Zhang, B. Sendhoff, and E. Tsang, "Combining model-based and genetics-based offspring generation for multi-objective optimization using a convergence criterion," in Proceedings of the 2006 IEEE International Conference on Evolutionary Computation, pp. 892-899, Vancouver, Canada, July 2006.

[31] L. C. Bezerra, M. López-Ibánez, and T. Stützle, “An empirical assessment of the properties of inverted generational distance on multi-and many-objective optimization," in Proceedings of the International Conference on Evolutionary Multi-Criterion Optimization, pp. 31-45, Munster, Germany, February 2017.

[32] H. Wang, Y. Jin, and X. Yao, "Diversity assessment in manyobjective optimization," IEEE Transactions on Cybernetics, vol. 47, no. 6, pp. 1510-1522, 2017.

[33] L. While, P. Hingston, L. Barone, and S. Huband, "A faster algorithm for calculating hypervolume," IEEE Transactions on Evolutionary Computation, vol. 10, no. 1, pp. 29-38, 2006.

[34] K. Deb and S. Jain, "Running performance metrics for evolutionary multi-objective optimizations," in Proceedings of the Fourth Asia-Pacific Conference on Simulated Evolution and Learning (SEAL'02), pp. 13-20, Singapore, 2002.

[35] Y.-N. Wang, L.-H. Wu, and X.-F. Yuan, "Multi-objective selfadaptive differential evolution with elitist archive and crowding entropy-based diversity measure," Soft Computing, vol. 14, no. 3, pp. 193-209, 2010.

[36] J. R. Schott, Fault Tolerant Design Using Single and Multicriteria Genetic Algorithm Optimization, Massachusetts Institute of Technology, Cambridge, MA, USA, 1995.

[37] N. Riquelme, C. Von Lücken, and B. Baran, "Performance metrics in multi-objective optimization," in Proceedings of the 2015 Latin American Computing Conference (CLEI), pp. 1-11, Arequipa, Peru, December 2015.

[38] Y. Tian, T. Zhang, J. Xiao, X. Zhang, and Y. Jin, "A coevolutionary framework for constrained multi-objective optimization problems," in Proceedings of the IEEE Transactions on Evolutionary Computation, CEC 2011, New Orleans, LA, USA, June 2011.

[39] Y. Tian, X. Zhang, R. Cheng, C. He, and Y. Jin, "Guiding evolutionary multiobjective optimization with generic front modeling," IEEE Transactions on Cybernetics, vol. 50, no. 3, pp. 1106-1119, 2020.

[40] L. Zhang, X. Wu, H. Zhao, F. Cheng, and Q. Liu, "Personalized recommendation in $\mathrm{P} 2 \mathrm{P}$ lending based on risk-return management: a multi-objective perspective," in Proceedings of the EEE Transactions on Big Data, p. 1, San Francisco, CA, USA, 2020.

[41] Y. Xichang and Z. Quanren, "Practical implementation of the SCADA+AGC/EDC system of the Hunan power pool in the central China power network," IEEE Transactions on Energy Conversion, vol. 9, no. 2, pp. 250-255, 1994. 